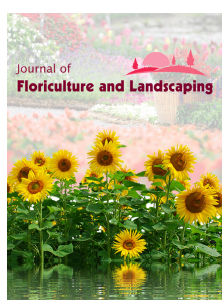

ISSN: $2663-6050$

Received: November 20, 2018 Accepted: December 28, 2018 Published: December 31, 2018

*Corresponding Author:

A. Arunesh

Email: arunesharasu@gmail.com

\section{Ethnobotanical studies of weed plants in rice field ecosystem}

\author{
A. Arunesh*, P. Karuppaiah, Ajish Muraleedharan \\ Department of Horticulture, Faculty of Agriculture, Annamalai University, Annamalainagar, Tamil Nadu, India
}

\begin{abstract}
Tamilnadu is one of the leading states in rice production in India. Weeds are widely found and are tremendously grown everywhere on paddy fields. Ethnobotany have gained importance during recent years. Based on this, an ethnobotanical exploration has been carried to find out the medicinal values of weed plants growing in the paddy fields of Annamalai Nagar, Cuddalore district. The study reveals the importance of the weed plants associated with paddy fields, a total of 40 species of weeds belonging to 22 families has been recorded in meeting the multiple requirements of human beings.
\end{abstract}

KEYWORDS: Rice, weeds, ethnobotany, medicinal values

\section{INTRODUCTION}

India is one among the major biodiversity rich countries of the world. Paddy (Oryza sativa L.) is a major food crop of the world and is cultivated widely in India. Ethnobotany is usually defined as anthropological approach to botany [1]. Thus, 'Ethno' refers to people, culture, language, knowledge and practice and 'Botany' is the study of plants. Weeds are very common in all parts of Tamil Nadu, India, and mainly in agricultural fields like rice fields [2]. Thus, the present study deals with ethnobotanical aspects of the major weeds of paddy fields in Tamilnadu.

\section{MATERIALS AND METHODS}

\section{Study area}

Annamalai Nagar is a special grade panchayat town in cuddalore district of Tamilnadu. It is located at about $6 \mathrm{Km}$ west of Bay of Bengal at $11^{\circ} 24^{\prime}$ North latitude and $79^{\circ} 41^{\prime}$ East longitude and at an altitude of $+5.79 \mathrm{M}$ above the Mean sea level (MSL). The climate is moderately warm with hot summer months. The mean maximum temperature ranges from $29.7^{\circ} \mathrm{C}$ to $38.3^{\circ} \mathrm{C}$ with a mean of $32.42^{\circ} \mathrm{C}$ while, the mean minimum temperature ranges from $21.1^{\circ} \mathrm{C}$ to $27.0^{\circ} \mathrm{C}$ with a mean of $24.1^{\circ} \mathrm{C}$. The mean relative humidity is $73 \%$.

\section{Methodology}

The ethnobotanical survey was conducted in the selected paddy fields of Annamalai Nagar region in cuddalore district of
Tamilnadu during the year 2018. Observation on morphology of the weed and their habitat were recorded. The ethnobotanical research regarding the medicinal utility of the common weeds were collected with the help of field labours and local community. They had indigenous knowledge about the weed plants growing in the paddy fields. However, relevant literature was also consulted on the basis of interviews in local language. Standard procedure was adopted for preparing herbarium sheets. All collected specimens were critically examined in the Herbarium for proper identification.

\section{RESULTS AND DISCUSSION}

The ethnobotanical information in the study area about the use of weed plants in paddy fields of Annamalai Nagar region was well documented. All the major weeds are reported to have medicinal properties. The different parts of the plant that are used for medicinal purposes such as leaves, shoots, roots, seeds, fruits, flowers etc. These parts contain some medicinal value used to cure specific diseases. Their botanical features and medicinal potential are given in the table as follows. Based on the survey, there are 40 species of weeds belonging to 22 families were recorded (Table 1, Figure 1). The dominant family Amaranthaceae having 6 species, followed by Euphorbiaceae, Solanaceae and Poaceae having 4 species each, Cyperaceae having 3 spp, Asteraceae, Lamiaceae, having 2 spp each and the other families sharing 1 spp each. The collection of information obtained from the ethnobotanical survey was similar to the investigation done by Dhanam and Elayaraj [3] in paddy fields of Villupuram district.

Copyright: $\odot 2018$ The authors. This article is open access and licensed under the terms of the Creative Commons Attribution License (http://creativecommons.org/licenses/by/4.0/) which permits unrestricted, use, distribution and reproduction in any medium, or format for any purpose, even commercially provided the work is properly cited. Attribution - You must give appropriate credit, provide a link to the license, and indicate if changes were made. 
Table 1: Ethnobotanical aspects of weed plants in rice field

\begin{tabular}{|c|c|c|c|c|c|}
\hline S.No & Botanical name & Common name & Family & Vernacular name & Medicinal uses \\
\hline 1 & Acalypha indica & Indian copperleaf & Euphorbiaceae & Kuppaimeni & Asthma, pneumonia, bronchitis \\
\hline 2 & Achyranthes aspera & Devil's horsewhip & Amaranthaceae & Naayuruvi & Kidney stone, leprosy, haemorrhoids \\
\hline 3 & Aerva lanata & Javanese wool plant & Amaranthaceae & Sirukkan poolai & Constipation, head ache, gonorrhoea \\
\hline 4 & Alternanthera sessilis & Sessile pigweed & Amaranthaceae & Ponnanganni & Stomach disorders, night blindness, \\
\hline 5 & Amaranthus spinosus & Spiny pigweed & Amaranthaceae & Mullu keerai & $\begin{array}{l}\text { Diarrhea, tooth ache, eczema, urinary } \\
\text { troubles }\end{array}$ \\
\hline 6 & Amaranthus viridis & Slender amaranth & Amaranthaceae & Kuppaikeerai & Dysentery, inflammations, constipation \\
\hline 7 & Bergia capensis & Cape ash & Elatinaceae & Neeru paavila & Against Intestinal worms, food poisoning \\
\hline 8 & Cardiospermum helicacabum & Balloon vine & Sapindaceae & Modakkanthan & Rheumatism, nervous diseases, ear ache \\
\hline 9 & Centella asiatica & Asiatic pennywort & Apiaceae & Vallaarai & $\begin{array}{l}\text { Increases memory, healing wounds, } \\
\text { atherosclerosis, asthma }\end{array}$ \\
\hline 10 & Chloris barbata & Purple chloris & Poaceae & Corai pillu & Diabetes, skin diseases, fever \\
\hline 11 & Cleome viscose & Tick weed & Cleomaceae & Vaelai keerai & Rheumatism, ulcer, ear ache, scurvy \\
\hline 12 & Commelina bhengalensis & Tropical spiderwort & Commelinaceae & Kaanaakuzhai & Dysentery, infertility, burns, rashes, leprosy \\
\hline 13 & Convolvulus arvensis & Field bindweed & Convolvulaceae & Bhoomi Chakra Poondu & Heal spider bites, Purgative \\
\hline 14 & Croton sparciflorus & Ban tulasi & Euphorbiaceae & Rail poondu & Malaria, cough, ulcer, hypertension \\
\hline 15 & Cynodon dactylon & Bermuda grass & Poaceae & Arugampul & $\begin{array}{l}\text { Leucoderma, bronchitis, piles, asthma, } \\
\text { tumors, skin diseases }\end{array}$ \\
\hline 16 & Cyperus difformis & Umbrella sedge & Cyperaceae & Vattakorai & $\begin{array}{l}\text { Vermifuge, Diuretic and Antibacterial } \\
\text { properties. }\end{array}$ \\
\hline 17 & Cyperus rotundus & Purple nut sedge & Cyperaceae & Koraipil & Pyresis, Stomach ache, diarrhea \\
\hline 18 & Cyperus iria & Flat sedge/Yellow sedge & Cyperaceae & Oosikorai & Amenorrhoea, rheumatism, diuretic \\
\hline 19 & Echinochloa crus-galli & Barnyard grass & Poaceae & Kudiraivali & $\begin{array}{l}\text { Carbuncles, Haemorrhages, Sores, Spleen } \\
\text { trouble, Cancer }\end{array}$ \\
\hline 20 & Echinochloa colona & Jungle rice & Poaceae & Sauri & $\begin{array}{l}\text { Spleen and Haemorrhage problems, } \\
\text { Antimicrobial activity }\end{array}$ \\
\hline 21 & Eclipta alba & False daisy & Asteraceae & Karisalaan kanni & $\begin{array}{l}\text { Diabetes, cirrhosis of liver, hepatitis, skin } \\
\text { diseases }\end{array}$ \\
\hline 22 & Euphorbia hirta & Asthma herb & Euphorbiaceae & Amman pacharisi & $\begin{array}{l}\text { Cancer, bronchial infections, abscesses, } \\
\text { asthma }\end{array}$ \\
\hline 23 & Gomphrena celosioides & Bachelor's button & Amaranthaceae & Neer vadamalli & Dermatitis, Asthma \\
\hline 24 & Heliotropium indicum & Indian heliotrope & Boraginaceae & Siruthael kodukku & $\begin{array}{l}\text { Warts, inflammations, tumors, skin } \\
\text { problems, ulcer }\end{array}$ \\
\hline 25 & Hygrophila auriculata & Marsh barbel & Acanthaceae & Neermuli & $\begin{array}{l}\text { Dysuria, spermatorrhoea, diabetes, } \\
\text { rheumatism }\end{array}$ \\
\hline 26 & Leucas aspera & Common leucas & Lamiaceae & Thumbai & $\begin{array}{l}\text { Wounds, sores, chronic skin diseases, } \\
\text { scabies, fever }\end{array}$ \\
\hline 27 & Marsilea quadrifolia & European water clover & Marsileaceae & Arakeerai & $\begin{array}{l}\text { Snakebites, anti inflammatory, diuretic, } \\
\text { skin injuries }\end{array}$ \\
\hline 28 & Mimosa pudica & Touch me not plant & Fabaceae & Thotta chinungi & Haemorrhoids, jaundice, wounds \\
\hline 29 & Ocimum canum & Hoary basil & Lamiaceae & Naaithulasi & $\begin{array}{l}\text { Fever, cough, diabetes, dysentery, tooth } \\
\text { ache }\end{array}$ \\
\hline 30 & Phyla nodiflora & Frog fruit & Verbinaceae & Poduthalai & $\begin{array}{l}\text { Ulcers, wounds, burns and boils, gastric } \\
\text { troubles }\end{array}$ \\
\hline 31 & Phyllanthus niruri & Gale of wind & Euphorbiaceae & Keela nelli & Jaundice, stomach pain, ulcer, fever \\
\hline 32 & Physalis minima & Sunberry/Wild gooseberry & Solanaceae & Tholthakkaali & $\begin{array}{l}\text { Urinary purgative, diuretic, itching, } \\
\text { hypertension }\end{array}$ \\
\hline 33 & Portulaca oleracea & Common purslane & Portulacaceae & Pasalai & $\begin{array}{l}\text { Cough, dysentery, burns, skin diseases, } \\
\text { dyspepsia }\end{array}$ \\
\hline 34 & Sida acuta & Common wire weed & Malvaceae & Arivaalmunai poondu & Swelling, indigestion, blood clot, head ache \\
\hline 35 & Solanum nigrum & Black nightshade & Solanaceae & Manathakkaali & $\begin{array}{l}\text { Cancerous sores, boils, leucoderma, } \\
\text { stomach pain, ulcers, rabies, ring worm }\end{array}$ \\
\hline 36 & Solanum trilobatum & Purple eggplant & Solanaceae & Thoodhuvalai & Cold, cough, asthma, rheumatism, leprosy \\
\hline 37 & Solanum xanthocarpum & Yellow berried nightshade & Solanaceae & Kandan kathiri & Bronchitis, cough, constipation, asthma \\
\hline 38 & Sphenoclea Zeylanica & Goose weed & Sphenocleacee & Kopuram thaangi & Ulcers \\
\hline 39 & Tribulus terrestris & Puncture vine & Zygophyllaceae & Nerinji & Diabetes, crystalluria, cholesterol, diuretic \\
\hline 40 & Tridax procumbens & Coat buttons & Asteraceae & Vettukaya poondu & $\begin{array}{l}\text { Diarrhoea Arrests bleeding, antiseptic, } \\
\text { ulcer }\end{array}$ \\
\hline
\end{tabular}

Ethnobotanical studies are helpful in revealing medicinal potential of underutilized plants especially in complicated and minor diseases [4]. Many plants and their products recently used in industries were developed through the indigenous ethnobotanical knowledge. So, it is need of the time to enhance our ethnobotanical knowledge on weeds plants. 


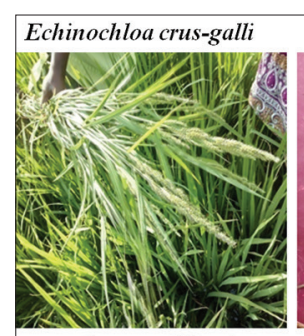

Cyperus difformis

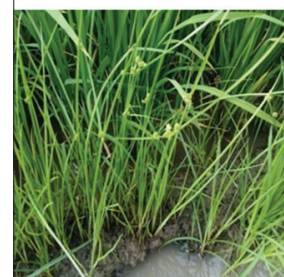

Sphenoclea Zeylanica
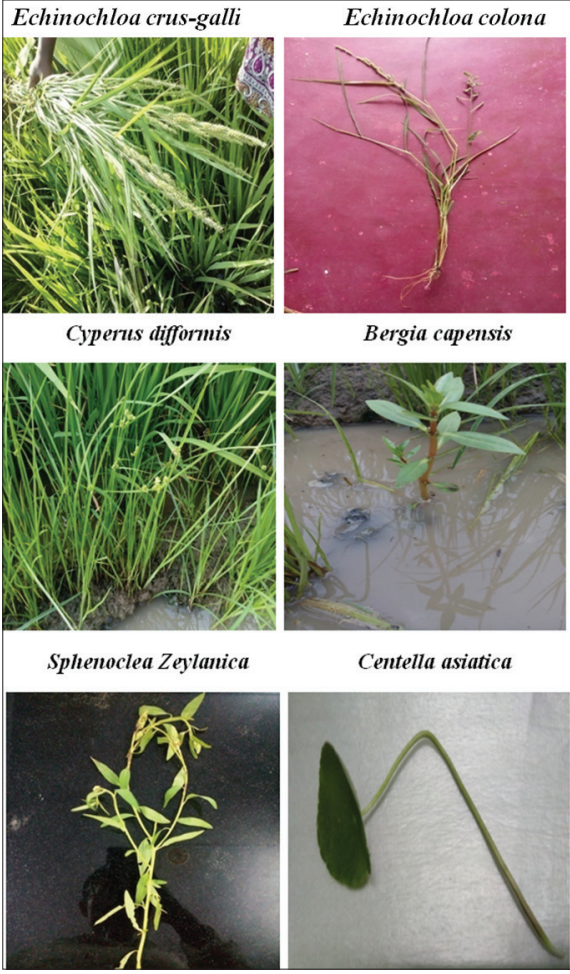

Bergia capensis

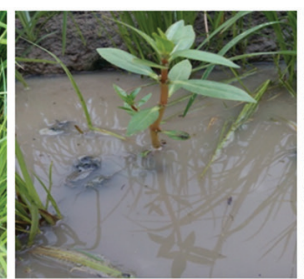

Centella asiatica

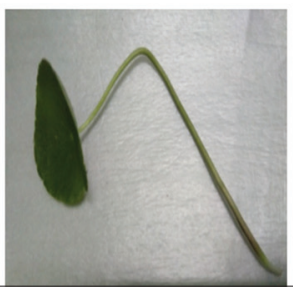

Figure 1: Major weeds present in rice fields
But modernization resulted in decrease of ethnobotanical information and hence attempts are being made to make a permanent record on plants and their uses by Botanical Authorities of India [5]. Thus in this pollution rich environment human beings are prone to diseased conditions easily and these ethnobotanical aspects are dealing it a readymade solution to solve those problems especially in rural regions. There is a need of the hour to investigate these studies further and explore more things.

\section{REFERENCE}

1. Jain SK, Jain A. Indian Ethnobotany-Bibliography of $21^{\text {st }}$ Century (2001-2015). Scientific Publishers; 2016 Jun 1.

2. Parameswaran P, Kumar A. An account of the 'useful weeds' associated with wetland paddy fields (Vayals) of wayanad, Kerala, India. Annals of Plant Sciences. 2016;6(01):1516-26.

3. Dhanam S, Elayaraj B. Ethnomedicinal aspects of some weeds from paddy fields of Villupuram district in Tamil Nadu, India. International Letters of Natural Sciences. 2014;14:1-10.

4. Qureshi R, Ghazanfar SA, Obied H, Vasileva V, Tariq MA. Ethnobotany: A living science for alleviating human suffering. Evidence-Based Complementary and Alternative Medicine. 2016;2016. DOI: http:// dx.doi.org/10.1155/2016/9641692

5. Lal HS, Singh S, Priya K. Study of ethno medicinal uses of weeds in rice field of Hazaribag district of Jharkhand, India. International Journal of Integrative sciences, Innovation and Technology. $2012 ; 1(2): 23-6$. 